\title{
LncRNA NCK1-AS1 promotes proliferation and induces cell cycle progression by crosstalk NCK1-AS1/miR-6857/CDK1 pathway
}

\author{
Haiyu Li ${ }^{1}$, Yongqin Jia ${ }^{1,2}$, Junning Cheng ${ }^{1,3}$, Geli Liu ${ }^{1,2}$ and Fangzhou Song ${ }^{1,2}$
}

\begin{abstract}
The purpose of this study was to develop an IncRNA signature to improve the prediction of the prognosis of cervical cancer through integration bioinformatics and analysis of TCGA RNA sequencing data. In this study, we established a set of four IncRNA signatures that was significantly associated with recurrence-free survival using the Cox regression model. Functionally, we screened the CC-associated IncRNA NCK1-AS1 as a new candidate IncRNA and regulator which promotes development and progression in CC. qRT-PCR and RNA in situ hybridization (RISH) results showed that NCK1-AS1 was significantly up-regulated in 77.4\% (24/31) of the CC tissue group compared with the normal group $(P<0.01)$. Interestingly, we demonstrated that transcription factor SP1 directly binds to the promoter to activate NCK1-AS1 expression in SiHa cells. In vitro and in vivo assays of silencing NCK1-AS1 significantly inhibited cell proliferation and invasion, with induction of cell arrest in S phase of the cell cycle. Furthermore, Human Transcriptome Array 2.0 analysis after NCK1-AS1 silencing highlighted alterations in cell proliferation and cell cycle pathways. NCK1AS1 functioned as a molecular sponge for miR-6857, antagonizing its ability to repress CDK1/6 protein translation. In conclusion, these findings suggest that NCK1-AS1/miR-6857/CDK1 crosstalk serve as a critical effector in cervical cancer progression and may serve as a potential target in cervical cancer.
\end{abstract}

\section{Introduction}

Cervical cancer is the fourth-most common cause of cancer and deaths of cancer in women worldwide ${ }^{1-3}$. Despite the great efforts that have been made on HPV vaccines to protect women from cervical cancer, this malignancy remains the second-most common cause of female-specific cancer after breast cancer ${ }^{4}$. Nearly $80 \%$ of cervical cancers occur in developing countries. Squamous cell cancer is the cervical cancer with the greatest

\footnotetext{
Correspondence: Fangzhou Song (fzsongcq@163.com)

${ }^{1}$ Molecular Medicine and Cancer Research Center, Chongqing Medical University, Chongqing 400016, China

${ }^{2}$ Department of Biochemistry and Molecular Biology, Chongqing Medical University, Chongqing 400016, China

Full list of author information is available at the end of the article Edited by G. Calin
}

incidence. Unfortunately, there are no definite diagnostic and prognostic biomarkers ${ }^{5,6}$.

Increasing evidence demonstrates that long non-coding RNA (lncRNA) expression is tissue-specific and frequently dysregulated in various types of cancers,and some lncRNAs are correlated with cancer recurrence and poor prognosis $^{7-9}$. LncRNAs are defined as non-protein coding transcripts longer than 200 nucleotides, and were initially thought to represent spurious transcriptional noise. In general, the majority ( 78\%) of lncRNAs are characterized as tissue-specific, as opposed by only $\sim 19 \%$ of mRNAs. In addition to higher tissue specificity ${ }^{8,10-12}$, IncRNAs are characterized by higher developmental stage specificity, and cell subtype specificity in heterogeneous tissues, such as human neocortex ${ }^{13,14}$. Large-scale sequencing of cDNA libraries and more recently transcriptomic sequencing by 
Table 1 Four IncRNAs were significantly associated with the CC survival in the test data set

\begin{tabular}{|c|c|c|c|c|}
\hline Gnene symbole & Coefficient & Hazard ratio & $P$ value cox & $P$ value permutation \\
\hline AATBC & 0.7521141 & 0.471369 & $6.69 \mathrm{E}-04$ & 0.0004 \\
\hline NCK1-AS1 & 1.0531199 & 2.8665807 & $3.25 \mathrm{E}-05$ & 0.00234 \\
\hline LINC00937 & -1.0104329 & 0.3640613 & 3.06E-05 & 0 \\
\hline LINC00173 & -0.4792615 & 0.6192405 & $1.03 \mathrm{E}-02$ & 0.0064 \\
\hline
\end{tabular}

next generation sequencing indicate that long noncoding RNAs number are in the order of tens of thousands in mammals ${ }^{15-17}$. However, despite accumulating evidence suggesting that the majority of these are likely to be functional, only a relatively small proportion has been demonstrated to be biologically relevant. HOTAIR originates from the $\mathrm{HOXC}$, which represses transcription across $40 \mathrm{~kb}$ of the HOXD locus by altering chromatin trimethylation state. To achieve this, HOTAIR directs the action of Polycomb chromatin remodeling complexes in trans to govern the cells' epigenetic state and subsequent gene expression ${ }^{18-20}$. Components of the Polycomb complex $^{21}$, including Suz12, EZH2, and EED, contain RNA binding domains that may potentially bind HOTAIR and probably other similar ncRNA ${ }^{22-25}$. Recent evidence has raised the possibility that transcription of genes that escape from $\mathrm{X}$-inactivation might be mediated by expression of lncRNA within the escaping chromosomal domains ${ }^{26,27}$. The ability of lncRNAs regulating associated protein-coding genes may contribute to disease if misexpression of an lncRNA deregulates a protein coding gene with clinical significance. In a similar manner, an antisense lncRNA that regulates the expression of the sense BACE1 gene, a crucial enzyme in Alzheimer's disease etiology, exhibits elevated expression in several regions of the brain in individuals with Alzheimer's disease $\mathrm{e}^{28,29}$.

Previous studies provide several that lncRNAs are involved in CC progression, such as lncRNA-EBIC, TI10124, TI18382, TI21327, TI18318, TI22687, TI09485, and ASK00420. LncRNA-EBIC was founded to be an oncogenic lncRNA, which could promote tumor cell invasion in $\mathrm{CC}$ by binding to $\mathrm{EZH} 2$ and inhibiting $\mathrm{E}$ cadherin expression. There are still exists a large number of previously unexplored lncRNA alterations in CC, especially in the expression patterns of CC-specific lncRNAs ${ }^{30}$. To investigate aberrantly expressed lncRNAs in cervical cancer, we integrated bioinformatics and analysis of TCGA RNA sequencing data (13 normals and 306 cervical squamous cell carcinomas and endocervical adenocarcinomas). Using the Cox regression model, we identified a prognostic four-lncRNA signature from TCGA data. In this comprehensive characterization of aberrantly expressed lncRNAs, we identified a CC specific upregulated lncRNA named as NCK1-AS1, which is a new candidate lncRNA promoting development of CC; and its biological role and molecular mechanism were evaluated as well.

\section{Results}

Identification of CC-specific and prognostic IncRNAs from TCGA CESC data

To detect prognostic lncRNAs that involved in cervical cancer progression, we subjected analysis of TCGA cervical squamous cell carcinoma and endocervical adenocarcinoma RNA sequencing data by univariable Cox proportional hazards regression analysis. A set of fourlncRNA signatures were significantly correlated with patients' survival $(P<0.0001$; Table 1$)$. Based on the expression of these four lncRNAs for DFS prediction, established a risk-score formula as follows: Risk score $=$ (0.7521141 $\times$ expression level of AATBC) + (1.0531199 $\times$ expression level of NCK1-AS1)+ (1.0104329 $\times$ expression level of LINC00937) + ( $-0.4792615 \times$ expression level of LINC00173) Then, the four-lncRNA expression signature risk score for each patient in the training set were calculated. Patients were divided into low-risk $(n=50)$ and high-risk $(n=50)$ groups according to the median risk score as cut-off in the training set. As shown in Fig. 1a patients in the high-risk group had significantly shorter survival times than those in the low-risk group $(P<0.0001)$. To confirm the riskscore formula, the risk scores for another 120 patients in testing sets were calculated. Consistent with the abovedescribed result, patients in the high-risk group had significantly shorter survival times than those in the low-risk group $(P=0.0132)$ (Fig. 1b).

\section{GO and pathway analysis of four-IncRNA signature associated biological processes in $\mathrm{CC}$}

Gene Ontology (GO) functional enrichment analysis was performed to identify associated biological processes and signaling pathways according to the risk score for classification. These results were visualized as interaction networks with Cytoscape. Four-lncRNA were mainly enriched in GO terms related to biological processes (BP) 


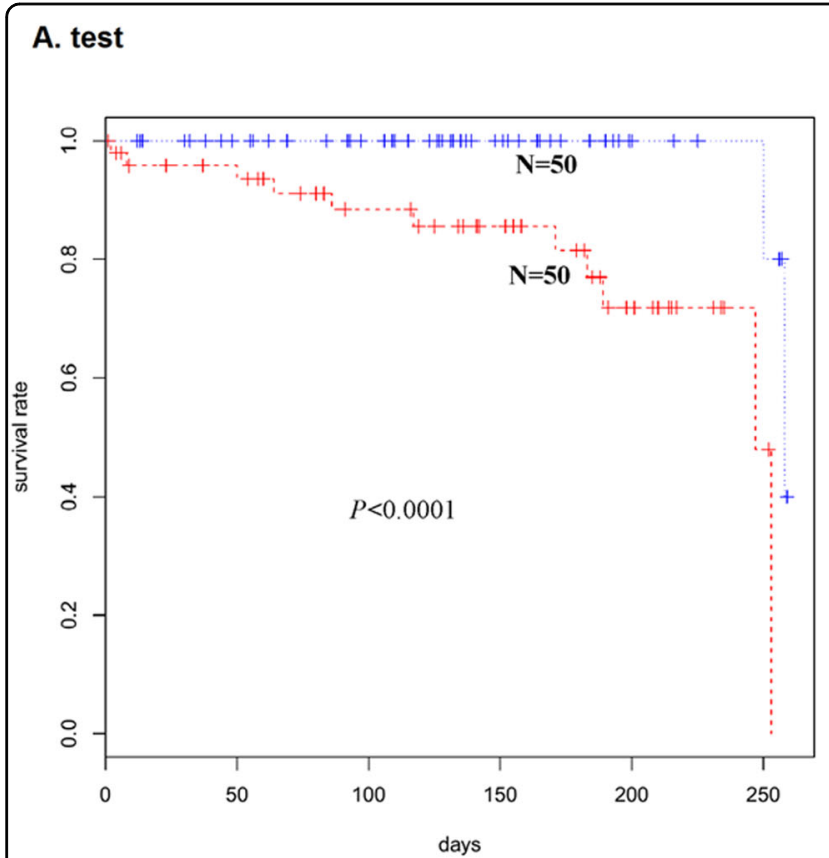

\section{B. validation}

Fig. 1 Kaplan-Meier curve of the cervical cancer survival from TCGA patients using the four-IncRNA risk-score formula. a Kaplan-Meier curves for test series $(N=100)$; b Kaplan-Meier curves for validation series $(N=120)$

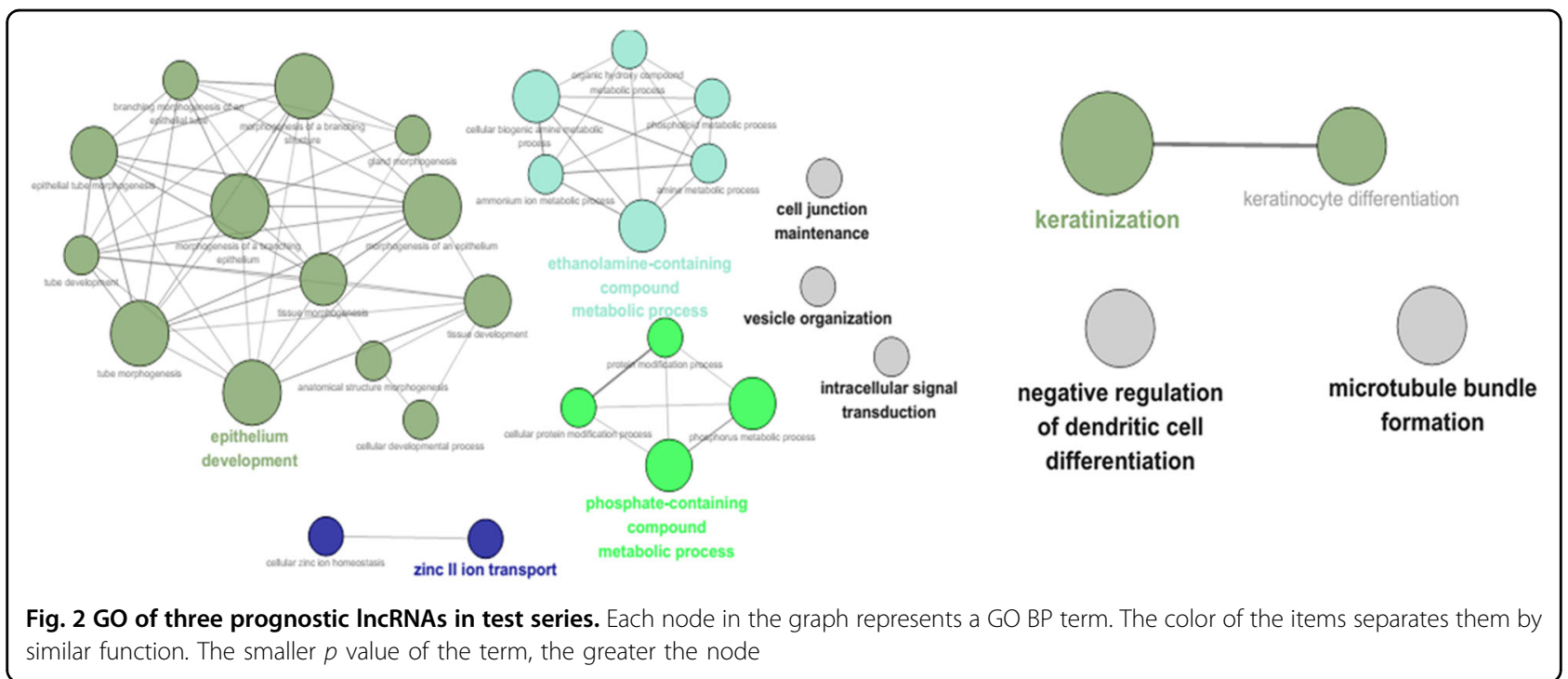

such as epithenlium development, intracellular signal transduction, cell junction maintenance microtubule bundle formation, negative regulation of dendritic cell differentiation and keratinization (Fig. 2).

\section{LncRNA NCK1-AS1 is specifically up-regulated in cervical cancer and associate with clinical progression}

Furthermore, we analyzed the expression of NCK1-AS1 and NCK1 in five cancers (Thyroid carcinoma, Kidney Chromophobe, Adrenocortical carcinoma, Breast invasive

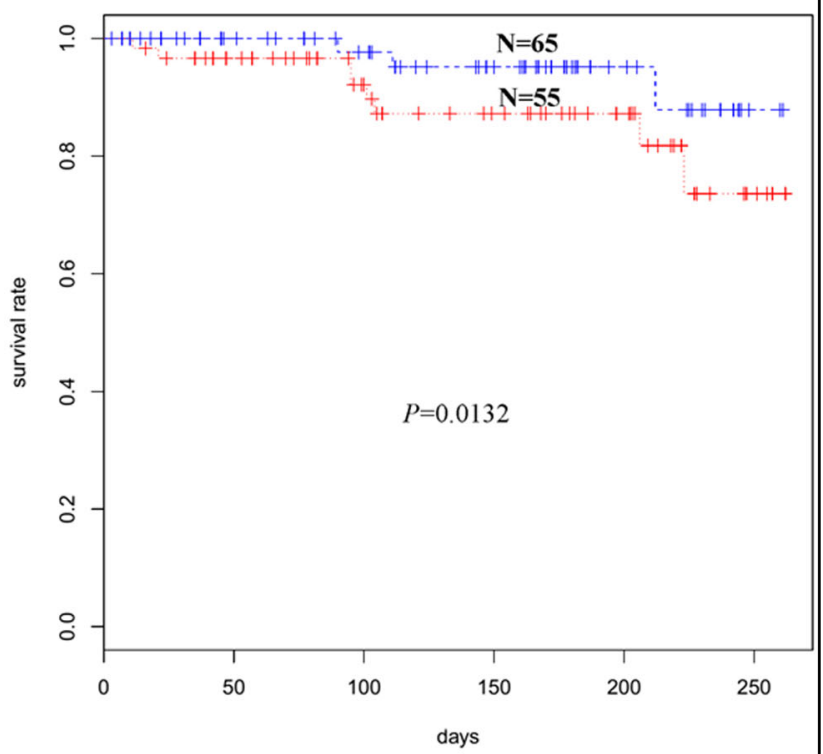

days 
performed to detect the level of NCK1-AS1 in 31 paired $\mathrm{CC}$ tissues and adjacent cancer normal tissues. Results confirmed that NCK1-AS1 was highly up-regulated $(P<$ $0.01)$ in $77.4 \%(24 / 31)$ of the CC tissues compared with the normal tissues (Fig. 3b, c). We also examined the expression of NCK1-AS1 in normal human cervical epithelial cells (HCerEpiC), three low metastatic cervical cancer cell lines (HeLa, C33A, and $\mathrm{SiHa}$ ) and one highly metastatic cell line (CaSki). Higher expression level of NCK1-AS1 was observed in cervical cancer cell lines compared with the human cervical epithelial cells. The highest level of NCK1-AS1 was in the metastatic cancer cell lines CaSki (Fig. 3d).

To investigate the relationship between NCK1-AS1 expression and cervical cancer clinical features, 31 patients were divided into high and low NCK1-AS1 expression level groups based on the median value. Statistical analysis showed no correlations between NCK1AS1 expression and age, lymph node numbers, tumor size, or clinical stage $(P>0.05)$. Interestingly, the NCK1AS1 expression level was significantly associated with histological type $(P<0.05)$ and lymph node status $(P<$ 0.05) (Table 2).

\section{Identification of NCK1-AS1 promoter region and transcription factor binding sites}

To identify the core promoter region of the NCK1-AS1 gene, four varieties of luciferase reporter constructs containing the overlapped different fragments of NCK1-AS1 gene $1000 \mathrm{bp}$ region upstream were constructed, as flowing D1000 (-1000-0), D750 (-750-0), D500 $(-500-0)$, and D250 (-250-0) (Fig. 4a). These four luciferase reporter constructs were transfected into $\mathrm{SiHa}$ cells and their luciferase activities were measured after $48 \mathrm{~h}$. Dual-Luciferase assay showed that luciferase activities were significant increased in cells transfected with D1000, D750, D500, and D250 compared with the pGL3-basic group. These data demonstrated that a genomic region from-750 to-250 of the NCK1-AS1 gene has a strong promoter activity (Fig. 4b). To further investigate the potential regulators involved in NCK1-AS1 over-expression, potential transcription factor binding sites in the NCK1-AS1 promoter were identified by JASPAR (http:// jaspar.genereg.net/). As shown in Fig. 4c, E2F1, XBP1, and SP1 binding sites were found in the promoter region of NCK1-AS1. The correlation between the NCK1-AS1 expression and these transcription factors E2F1, XBP1 and SP1 were performed and correlation analysis revealed that NCK1-AS1 has a significantly positive correlation with SP1 in TCGA CESC Tumor data set (Fig. 4d and Supplementary Fig.1C and 1D). SP1 over-expression promoted NCK1-AS1 expression in SiHa and CaSki cells, while up-regulation of E2F1 or XBP1 had no effect on NCK1-AS1 expression (Fig. 4e and Supplementary
Fig.1E). To ask whether SP1 directly transactivates NCK1AS1 expression, ChIP assay was performed. The result demonstrated that SP1 directly bound to the NCK1-AS1 promoter region (Fig. 4f). Furthermore, luciferase report assays indicated that SP1 binds to the D750 and D500 binding site, but not the D250 site (Supplementary Fig. 1F).

\section{NCK1-AS1 affects cell proliferation, migration and induces} cell cycle progression in vitro

To validate the function of NCK1-AS1 in regulating cervical cancer cell phenotype, knockdown of NCK1-AS1 in CaSki and SiHa cells that with high NCK1-AS1 expression was carried out via siRNA/shRNA mediated silencing and cell cycle profile and proliferation were subsequently analyzed. qRT-PCR analysis confirmed that the NCK1-AS1 expression level were significantly knocked down in two cell lines, (Supplementary Fig. 2A). qRT-PCR and western blot showed NCK1 expression level did unchanged after silencing of NCK1-AS1 (Supplementary Fig. 2b, c). As shown in Fig. 5a, CCK-8 assays showed that silencing of NCK1-AS1 significantly inhibited CC cell proliferation in vitro. Colony formation assay revealed that stable knockdown of NCK1-AS1 dramatically inhibited anchorage-independent growth abilities in CaSki, as the number and the size of formed colonies of the knockdown NCK1-AS1 cells were far fewer and smaller than those of control cells (Fig. 5b). Transwell assays showed that knockdown of NCK1-AS1 dramatically decreased cell invasion in CaSki and SiHa cells (Fig. 5c). Moreover, flow cytometry (FCM) and EdU immunofluorescent stain were performed to determine if NCK1-AS1 was involved in cell cycle regulation. The percentages of cells were significant increased in G1phase but decreased in S-phase in CaSki and SiHa cells with NCK1-AS1 knockdown (Fig. 5d). Consistently, EdU stain incorporation assay confirmed this result. Less EdUpositive cells with newly synthesized DNA (15\% and 17\% respectively) were detected in CaSki and $\mathrm{SiHa}$ cells with NCK1-AS1 knockdown compared with that in CaSki and SiHa control cells (38\% and 48\%, respectively, Fig. 5e, f). These data demonstrated that NCK1-AS1 affected the G1-S transition of cell-cycle progression and inhibited the proliferation, migration and invasion of $\mathrm{CC}$ cells.

\section{Depletion of NCK1-AS1 inhibits CC cell tumorigenesis in vivo}

To further validate the role of NCK1-AS1 in the tumorigenesis of cervical cancer, NCK1-AS1 stable knockdown CaSki cells or control cells were injected into nude mice. The result showed knockdown of NCK1-AS1 expression dramatically inhibited the tumor growth in both weight and size in nude mice (Fig. 6a, b). At the end of this experiment, tumor weight of NCK1-AS1 stable 


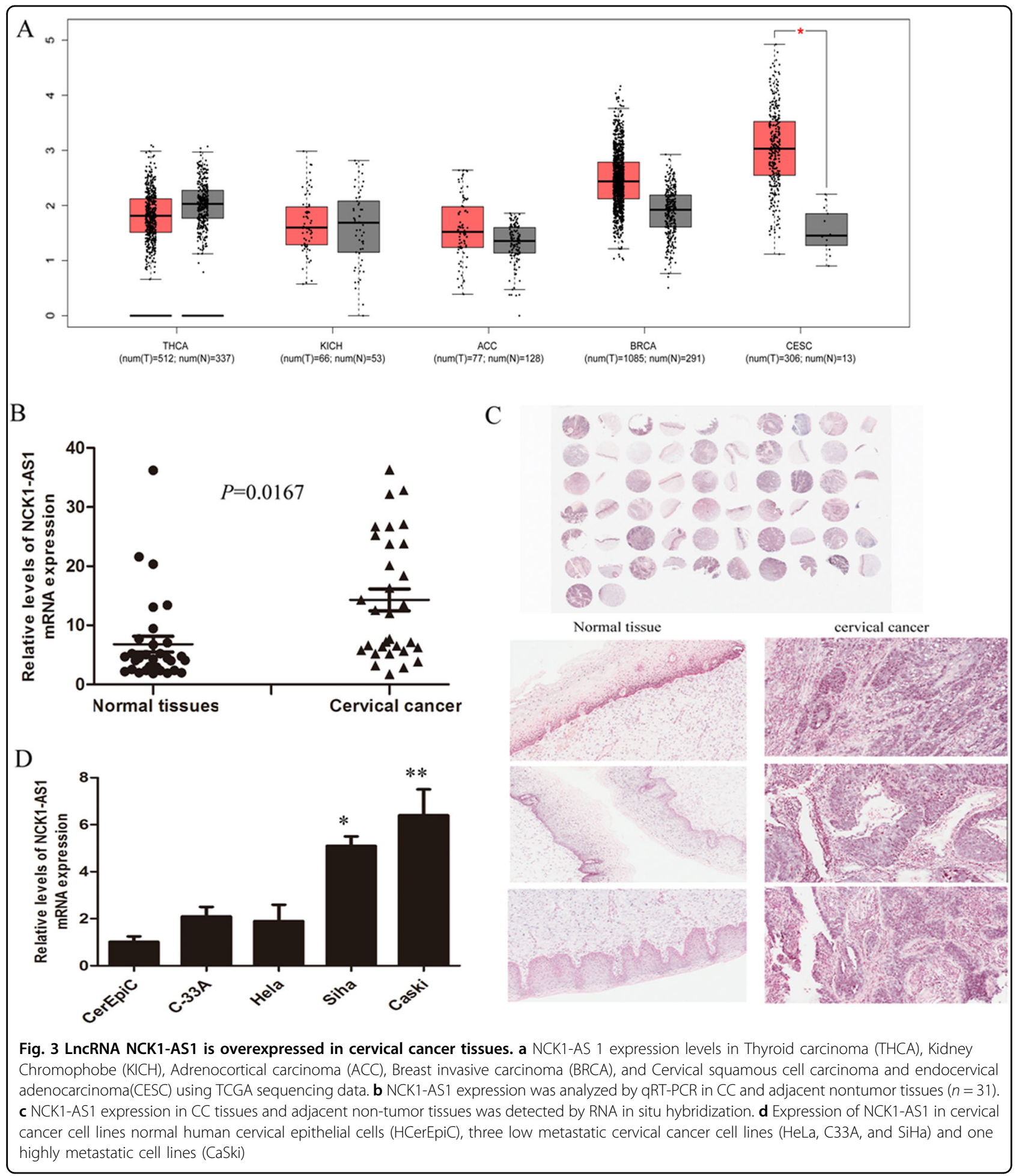

knockdown group $(0.683 \pm 0.121 \mathrm{~g})$ was only $7 \%$ of the control group $(0.053 \pm 0.015 \mathrm{~g})$ (Fig. 6c). Moreover, immunohistochemistry showed that tumor tissues of the NCK1-AS1 knockdown group had fewer Ki67-positive cells than the control group (Fig. 6d).

\section{Gene expression microarray analysis of NCK1-AS1} knockdown in CaSki cells

To further explore the potential molecular mechanisms of NCK1-AS1 in CC cells, Human Transcriptome Array 2.0 analysis was performed to investigate the differential 
Table 2 Relationship between NCK1-AS1 expression and clinicopathological factors

\begin{tabular}{|c|c|c|c|}
\hline \multirow[t]{2}{*}{ Characteristic } & \multirow[b]{2}{*}{ High expression } & \multirow{2}{*}{$\begin{array}{l}\text { NCK1-AS } \\
\text { Low expression }\end{array}$} & \multirow[t]{2}{*}{$P$-value } \\
\hline & & & \\
\hline Age(year) & & & 0.175 \\
\hline$\geq 50$ & 7 & 7 & \\
\hline$<50$ & 10 & 7 & \\
\hline Lymph node numbers & & & 0.419 \\
\hline$\geq 15$ & 15 & 6 & \\
\hline$<15$ & 6 & 1 & \\
\hline Lymph node status & & & 0.0144 \\
\hline No & 18 & 3 & \\
\hline N1 & 4 & 3 & \\
\hline Histological type & & & $<0.0001$ \\
\hline malignant & 24 & 7 & \\
\hline normal & 5 & 26 & \\
\hline Tumor size(cm) & & & 0.644 \\
\hline$<3$ & 9 & 2 & \\
\hline$\geq 3$ & 15 & 3 & \\
\hline Clinical stage & & & 0.823 \\
\hline 1 & 3 & 1 & \\
\hline$\|$ & 19 & 3 & \\
\hline III & 4 & 1 & \\
\hline
\end{tabular}

gene expression profiles between the NCK1-AS1 knockdown group and the control group in CaSki cells. The microarray raw data used in this study have been submitted to National Center for Biotechnology Information (NCBI) Gene Expression Omnibus (GEO) and are accessible through series accession number GSE107171 (http://www.ncbi.nlm.nih.gov/geo/query/acc.cgi? acc $=$ GSE107171)

By bioinformatics' analysis, 493 coding-genes and 413 non-coding genes were differentially expressed under the condition of " $\mathrm{Q}<0.001$ and fold change $>1.3$ ". All differentially expressed genes are clustered in Fig. 7a, b. Among the differentially expressed 493 coding protein genes, 165 genes were up-regulated (ratio, $>2.0$ ), and 328 genes were down-regulated (ratio, <0.5); while 301 genes were upregulated (ratio, >2.0), and 112 genes were downregulated (ratio, <0.5) among differentially expressed 413 non-coding protein genes. The top twenty genes' expression signatures in knockdown NCK1-AS1 CaSki cells are shown in Supplementary Table 1. PathwayRelationNetwork showed that these coding protein genes are related to the MAPK signaling pathway, cell cycle and Pathways in cancer (Fig. 7c). The Gene Ontology (GO) functional and Kyoto Encyclopedia of Genes and
Genomes (KEGG) pathway enrichment analysis indicated the differentially expressed 493 coding protein genes were enriched in extracellular matrix organization, cellular response to hypoxia, cell junction assembly, cell proliferation and epidermis development (Fig. 7d, e).

\section{NCK1-AS1 functions as ceRNA and sponges of miR-6857 in CC cells}

Several recent epigenetical studies have suggested that lncRNAs may act as competing endogenous RNAs (ceRNAs) to interact with miRNAs and influence the expression of these miRNAs in cell cytoplasm. Therefore, RNA-fluorescence in situ hybridization (RNA-FISH) was performed to examine its distribution and subcellular fractionation and founded that NCK1-AS1 was distributed in the cytoplasm in CaSki, SiHa and HeLa cells (Fig. 8a). To investigate the underlying mechanism of NCK1-AS1 function, we analyzed the lncRNA NCK1AS1 sequence and founded miR-6857 and miR-8067 binding sites using the TargetScan prediction algorithm (http://www.targetscan.org/vert_71/) (Fig. 8b). Therefore, we hypothesized that NCK1-AS1 competed to bind with miR-6857 and miR-8067 as a miRNA sponge, and then regulated the expression of downstream targets in 


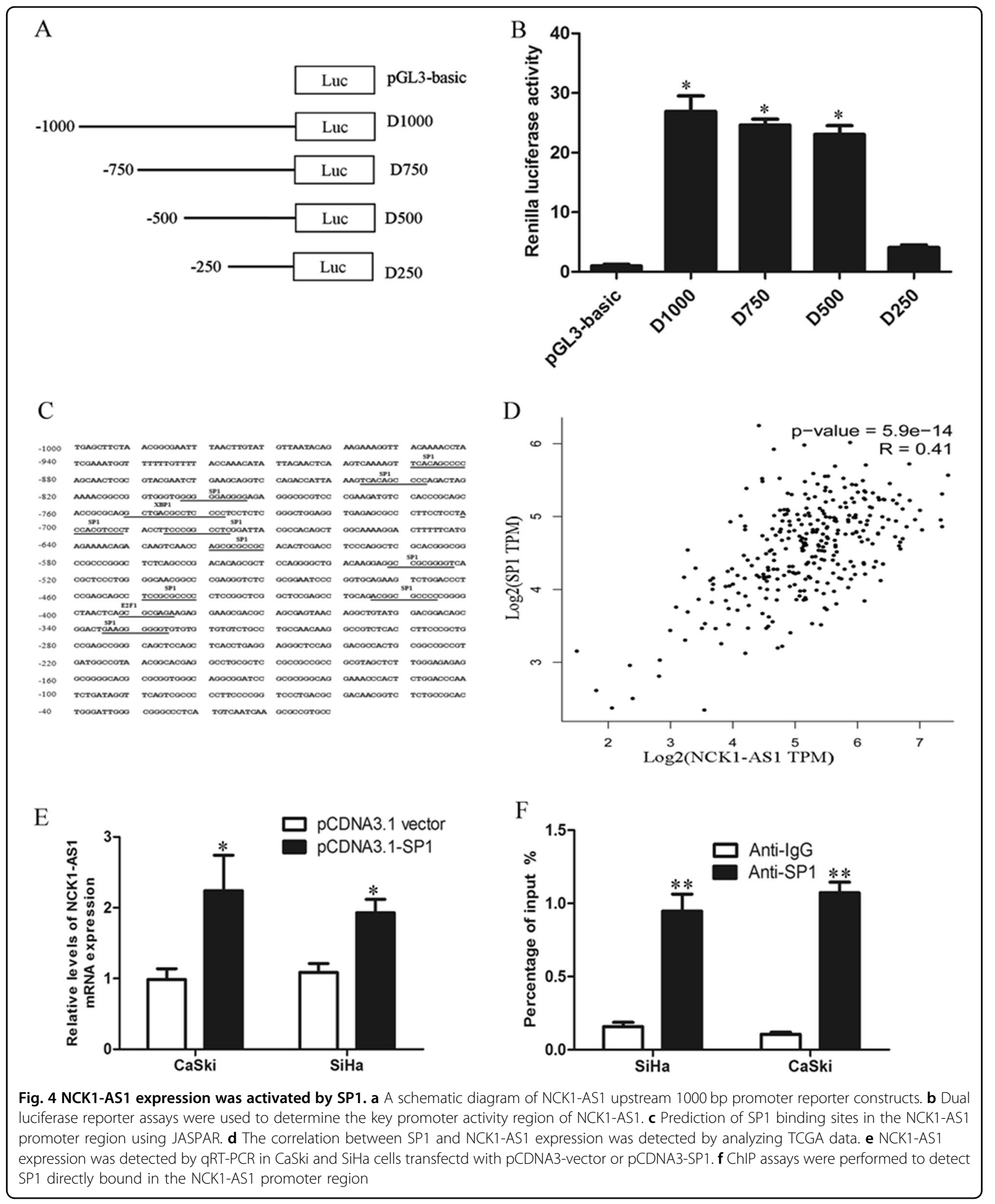

CC cells. Correction analysis showed that miR-6857 expression inversely correlated with NCK1-AS1 expressions in the TCGA cervical cancer data sets
(Supplementary Fig. 2D). qRT-PCR showed that knockdown of NCK1-AS1 dramatically increased the expression level of miR-6857, while NCK1-AS1 over-expression 


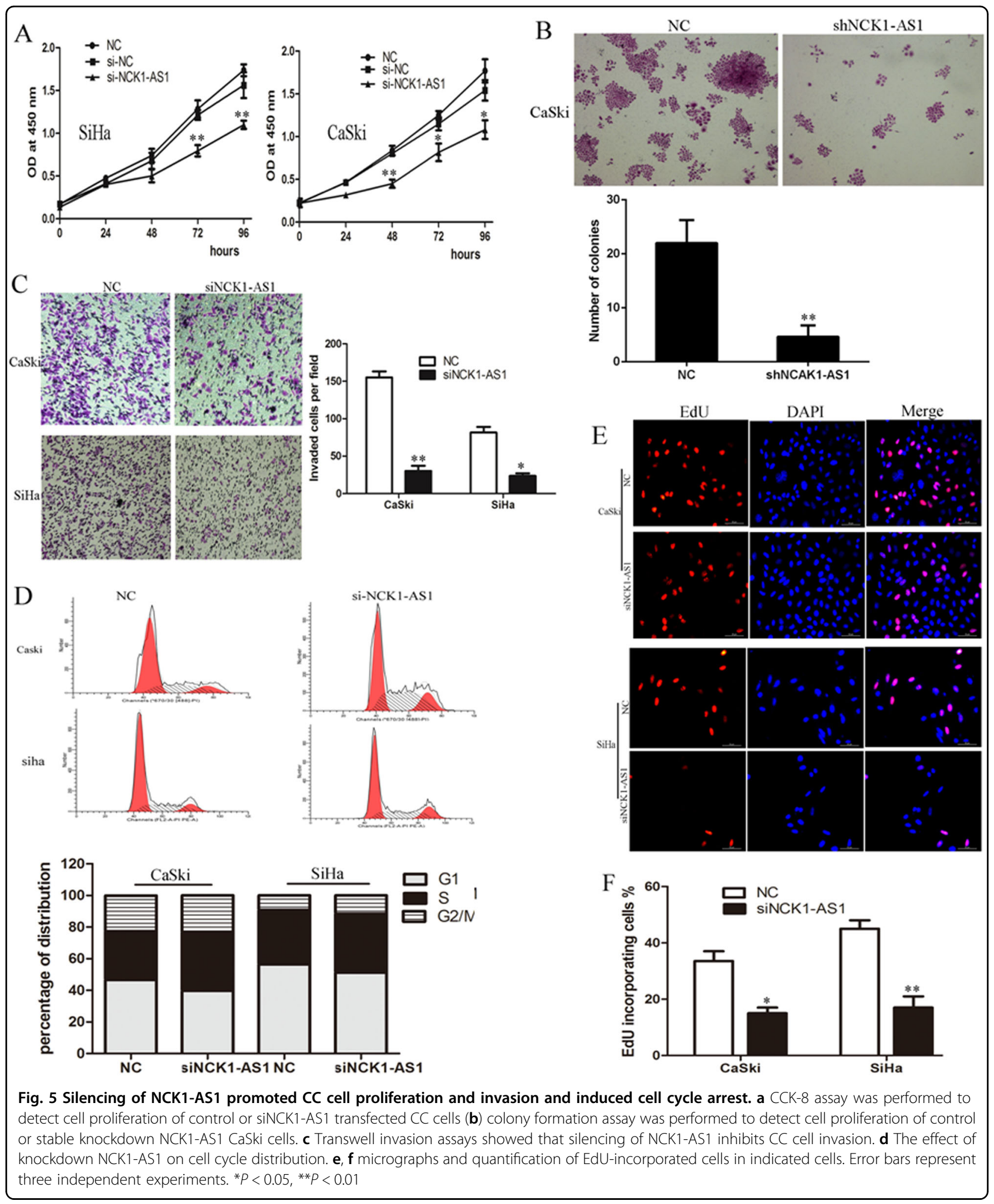

decreased the expression level of miR-6857, no change of the expression level of miR-8067 (Fig. 8b). For further confirmation, we constructed two dual-luciferase reporters containing: (1) Luc-NCK1-AS1-WT (2) LucNCK1-AS1 MT (mutated on the putative miR-6857 sites). As expected, over-expression of miR-6857 reduced the 


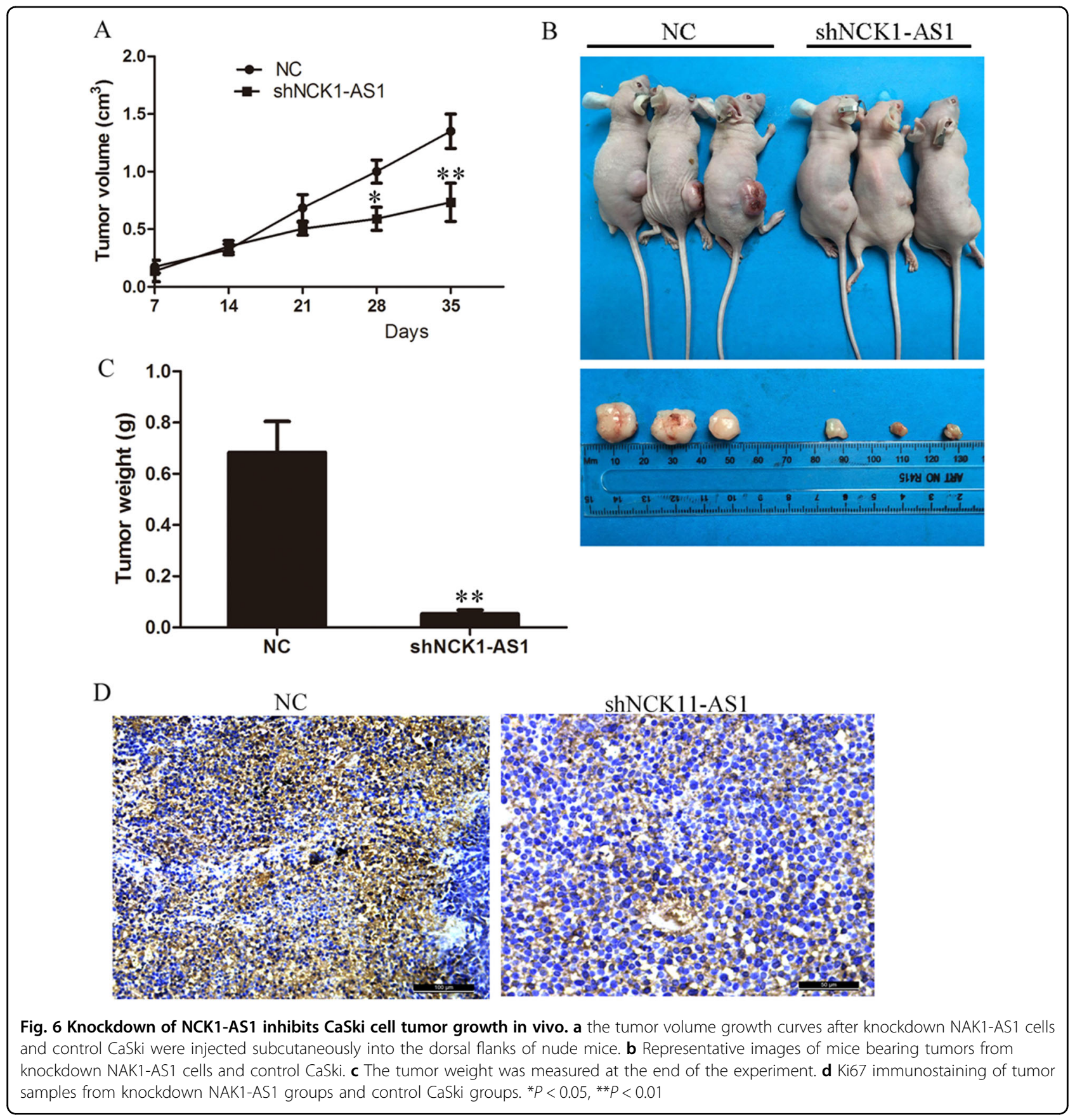

luciferase activities of the WT reporter vector but not empty vector or mutant reporter vector (Fig. 8c). Also, we wished to whether the exogenous over-expression of NCK1-AS1 induces a more malignant phenotype in cervical cancer. Structures of full-length and mutant (mutated on the putative miR-6857 sites) were generated (Supplementary Fig.3A). Hela cells constitutively GFP tagged full-length NCK1-AS1 (LV-NCK1-AS1), or GFPtagged NCK1-AS1 lacking putative miR-6857 binding sites (LV-NCK1-AS1 MT), (Supplementary Fig.3B). We next assessed the effects of NCK1-AS1 over-expression on invasion and cell proliferation. Results showed that LVNCK1-AS1 HeLa cells were significantly more invasive than both LV-NCK1-AS1 MT and wild type HeLa cells (Supplementary Fig.3C and D). Over-expression of fulllength NCK1-AS1 promoted the anchorage-independent growth of HeLa cells (Supplementary Fig.3E). However, over-expression of miR-6857 counteracted invasion and proliferation in LV-NCK1-AS1 HeLa cells (Supplementary Fig.3F). 


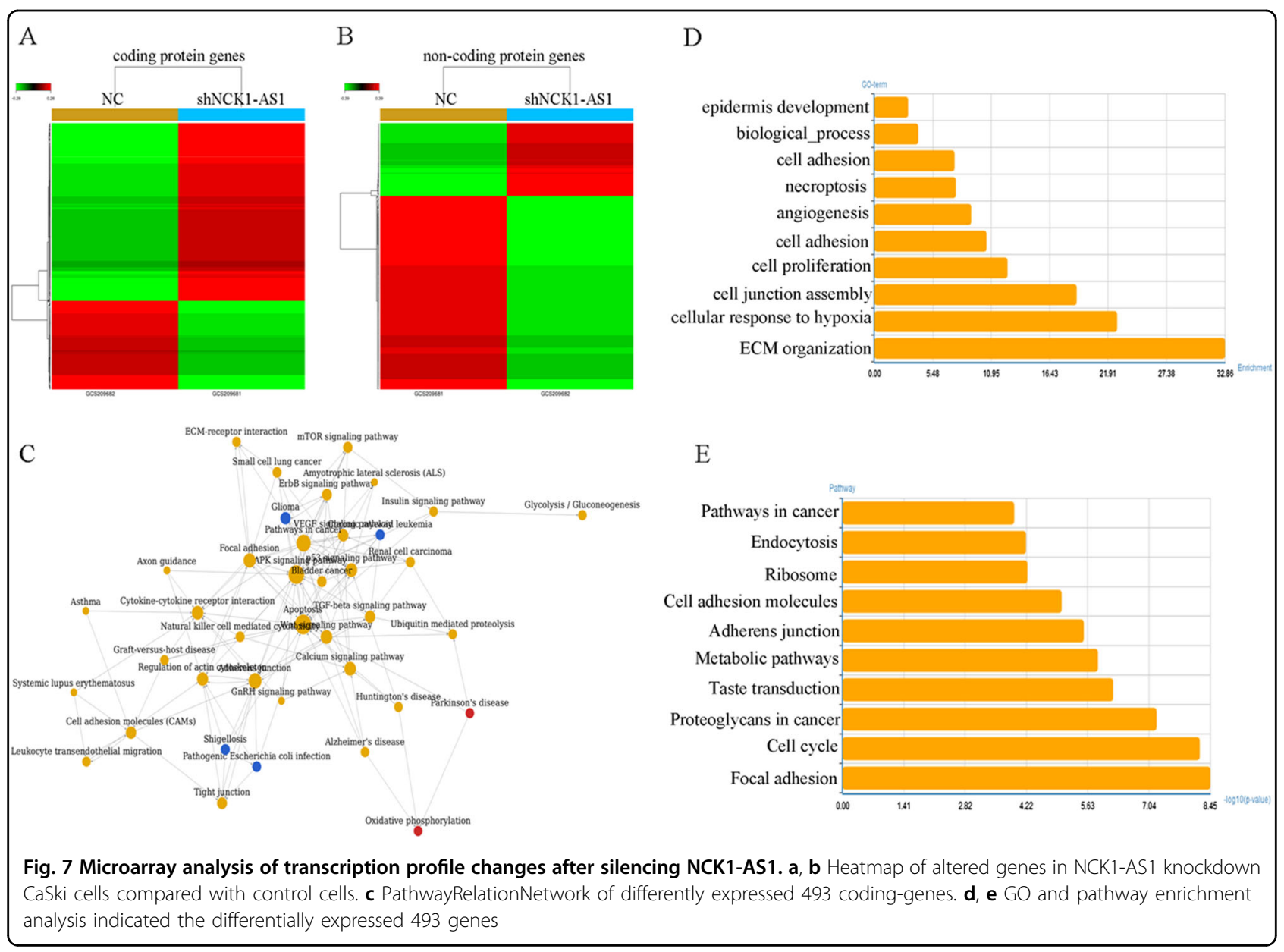

To understand the tumor suppressor function of miR6857, CCK8 and cell cycle assay were performed after miR-6857 control or mimics were transduced into the CaSki cells. As shown in Fig. 8d, miR-6857overexpressing CaSki cells displayed a significant increase in the percentages of cells in G2/M-phase but decreased proportions in S-phase cells. MiR-6857 overexpression inhibits the propagation of the $\mathrm{CC}$ cells as analyzed using CCK8 assay (Fig. 8e). Transwell assays were performed to detect the role of miR-6857 on invasion, and results showed that over-expression of miR6857 decreased CaSki cell invasion (Fig. 8f)

To address the mechanism underlying the suppressive effect of miR-6857 on the cell cycle of CC cells, we found that cell-cycle promoting genes, including CDK1/6, were examined as downstream targets of miR-6857 using TargetScan, a bioinformatic tool for miRNA target screening (Fig. 8g). Correlation analysis in CC tissues showed that NCK1-AS1 expression is positively associated with CDK1 expression, but not CDK6 (Fig. 8h). CDK1 is a catalytic subunit of the highly conserved protein kinase complex known as M-phase promoting factor
(MPF), which is essential for G1/S and G2/M phase transitions of eukaryotic cell cycle. We also found that a putative miR-6857 binding site on the 3-UTR of CDK1 was highly conserved in some species. Western blot assays also showed that knockdown of NCK1-AS1 decreased CDK1 protein levels, which is consistent with miR-6857induced down-regulation of CDK1 protein (Fig. 8i). Finally, we constructed luciferase reporters containing the putative miR-6857 binding sites, including wild-type (WT) or mutated miR-6857 binding sites. We found that over-expression of miR-6857 reduced the luciferase activities of the WT reporter vector but not mutant reporter vector (Fig. 8j, k).

In conclusion, these data indicate lncRNA NCK1-AS1 downregulated the RNA levels of miR-6857 through directly binding to them thereby derepressing CDK1 expression and imposing an additional level of posttranscriptional regulation (Fig. 9).

\section{Discussion}

Cervical cancer is both the fourth-most common cause of cancer and the fourth-most common cause of death 


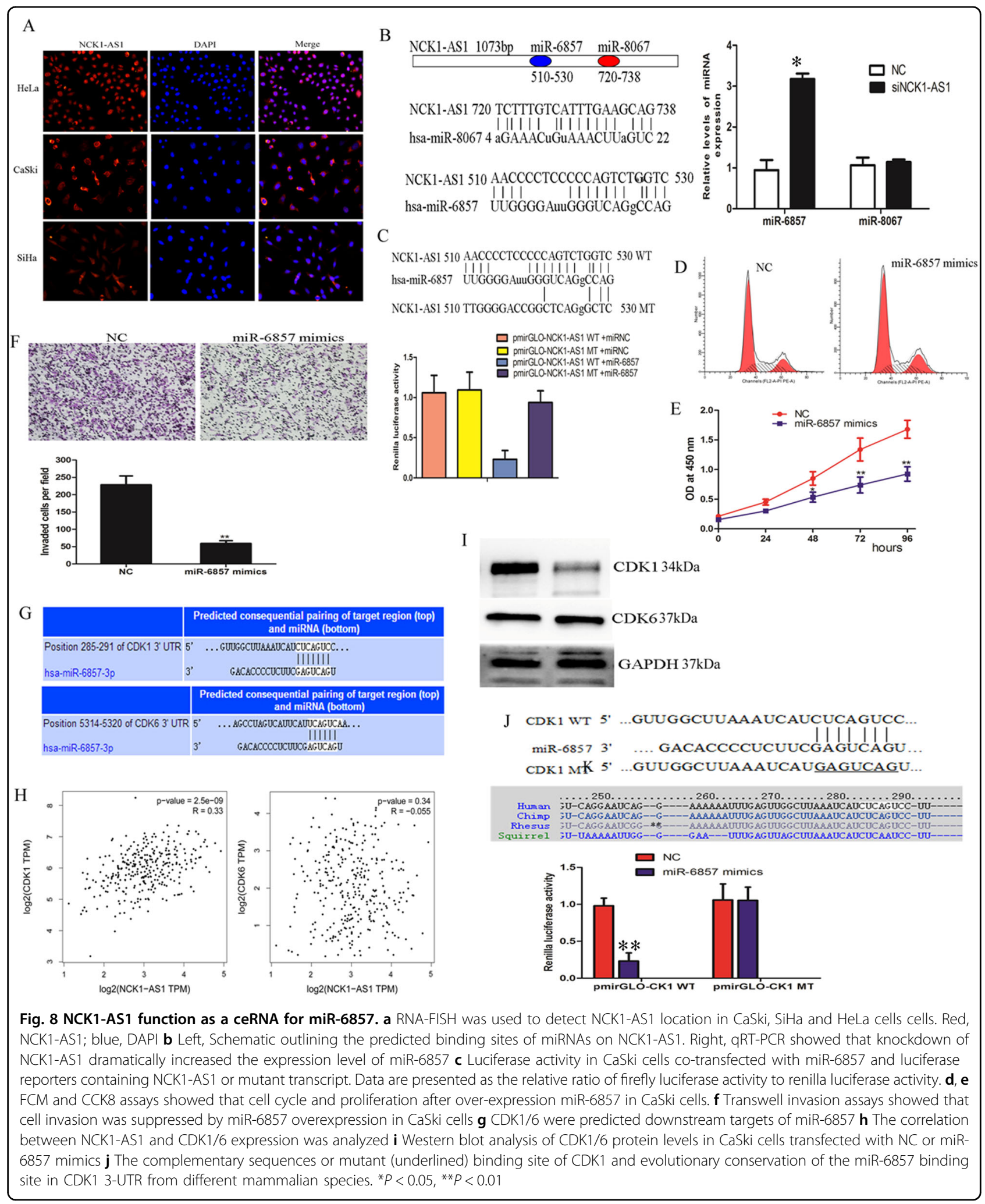

from cancer in women worldwide. In 2012, an estimated 528,000 cases of cervical cancer occurred, with 266,000 deaths. This is about $8 \%$ of the total cases and total deaths from cancer. About $70 \%$ of cervical cancers occur in developing countries ${ }^{5}$ and in low-income countries, it is the most common cause of cancer death ${ }^{1}$. Although 


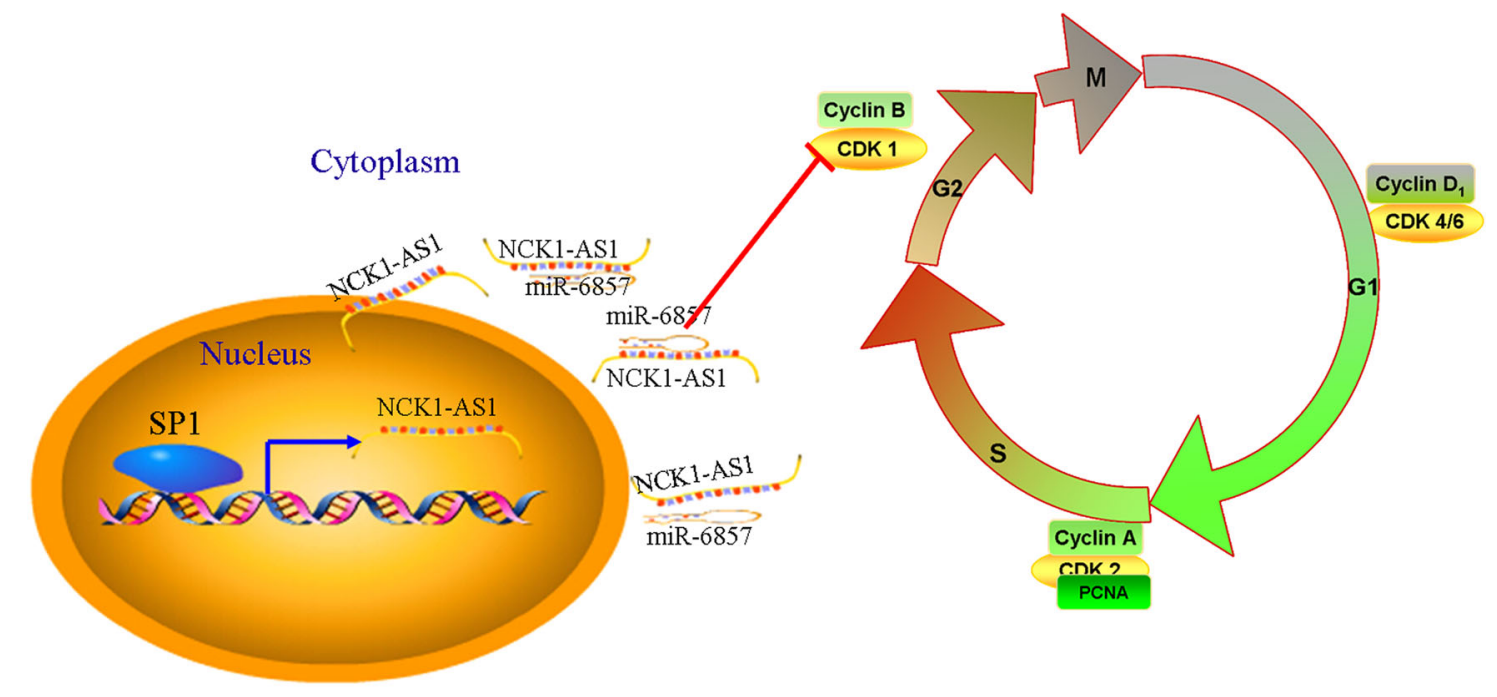

Fig. 9 Summary of the mechanism of NCK1-AS1 in CC cells

alterations of coding-protein genes in CC development are already a recognized phenomenon, little more than expression patterns of lncRNAs have been established. IncRNAs are a type of non-coding RNAs (ncRNAs) that exceed 200 nucleotides in length. IncRNAs are the relatively abundant component of the mammalian transcriptome and have been implicated in several cellular functions, including the regulation of gene transcription through the recruitment of chromatin-modifying enzymes $^{21,31,32}$. Increasing evidence suggests that these transcripts are frequently and aberrantly expressed in cancers, and some of them have been implicated in diagnosis and prognostication ${ }^{33,34}$.

In the present study, we examined the associations between the IncRNAs expression profile and clinical outcomes of $\mathrm{CC}$ patients using the sequencing data from TCGA project and identified a four-lncRNA signature that was significantly associated with the DFS. Based on our established risk-score formula, patients were divided into low-risk and high-risk groups according to the median risk score as cut-off in the training and testing set and patients in the high-risk group had significantly shorter median DFS than those in the low-risk group. We found that the IncRNA NCK1-AS1 has specific overexpression in cervical cancer tissue but not in other cancers. qRT-PCR and RISH result confirmed the NCK1AS1 expression pattern in CC clinical samples and NCK1AS1 expression was significantly associated with histological type and lymph node status. However, the transcription factors involved in NCK1-AS1 disorder expression in cervical cancer are not well known. In this study, our data demonstrated that SP1 directly bound to the NCK1-AS1 promoter region and activated NCK1-AS1 expression.
Previous studies demonstrated that lncRNAs play key roles in regulation of the malignant phenotypes of cancer cells. To further investigate the functions of lncRNA NCK1-AS1 in CC cells, we performed the loss-of-function evaluation in two CC cell lines (CaSki and $\mathrm{SiHa}$ ) with higher NCK1-AS1 expression. Our findings showed that knockdown of NCK1-AS1 inhibited CC cell proliferation and invasion, and induced cell cycle arrest in vitro. We also showed depletion of NCK1-AS1 inhibits CC cell tumorigenesis in vivo. RNA-fluorescence in situ hybridization (RNA-FISH) showed NCK1-AS1 was to be distributed in the cytoplasm. Human Transcriptome Array 2.0 analysis showed 493 coding-genes and non-coding genes were differentially expressed between NCK1-AS1 knockdown group and control group in CaSki cells. Moreover, we found NCK1-AS1 functions as a ceRNA for miR-6857 in the cytoplasm, and binding with miR-6857 releases its inhibition of CDK1/6 mRNA, resulting in elevated CDK1/6 protein levels.

Taken together, our study revealed that the GCassociated lncRNA NCK1-AS1 is an oncogenic lncRNA which promotes tumorigenesis through function as ceRNA for specific miRNAs. These data support the idea that crosstalk involving lncRNA NCK1-AS1/miR-6857/ CDK1 plays key roles in cervical cancer progression and potentially work as a therapy target.

\section{Materials and Methods}

\section{RNA sequence processing and IncRNA profile mining}

The level3 sequencing of cervical squamous cell carcinoma and endocervical adenocarcinoma and corresponding clinical data were downloaded from The Cancer Genome Atlas (TCGA) (https://cancergenome.nih.gov/). GATExplorer was used to process microarrays for gene 
expressions of lncRNAs. Gene expression data for other cancers (Kidney Chromophobe, Thyroid carcinoma, Adrenocortical carcinoma, Breast invasive carcinoma) were downloaded from the TCGA data set.

\section{LncRNA in situ Hybridization}

Surgical specimens of this study included tissues from 31 cervical squamous cell carcinoma and thire adjacent non-cancerous cervical squamous epithelium tissues were obtained from 31 patients who underwent potentially curative surgery in the First Affiliated Hospital of Chongqing Medical University from July 2013 to June 2015. The expression of NCK1-AS1 was examined by RISH in 31 pairs of paraffin-embedded tissues, which were cut into $4 \mu \mathrm{m}$ thick, and $2 \mathrm{~mm}$ diameter, sections to construct tissue microarrays (TMA). Briefly, the TMA were digested with proteinase $\mathrm{K}$, and hybridized with double digoxin-labeled LNA $^{\mathrm{TM}}$-modified NCK1-AS1 probe (Exiqon, Vedbaek, Denmark) overnight at $55^{\circ} \mathrm{C}$, then incubated overnight at $4 \mathrm{U}$ with an anti-DigoxigeninAP, Fab fragments (Roche, Basel, Switzerland 200:l). The cells nuclei were stained with NBT/BCIP (Roche, Basel, Switzerland) in the dark. Specific NCK1-AS1 ISH signals were identified as brown, punctate dots, and expression level was scored as Image-Pro Plus 6.0 software.

\section{Cells culture}

Human cervical epithelial cells (CerEpiC) from ScienCell Research Laboratories were cultured in Cervical Epithelial Cell Growth Supplement (CerEpiCGS, Cat \#7062), a complete medium designed for optimal growth of normal cervical epithelial cells in vitro. Four human cervical cancer cell lines ((HeLa, C33A and SiHa and CaSki) were obtained from Chinese Type Culture Collection, Chinese Academy of Sciences, were cultured in DMEM medium (Hyclone, Massachusetts, USA), and were supplemented with $10 \%$ fetal bovine serum (Hyclone, Massachusetts, USA), $100 \mathrm{U} / \mathrm{ml}$ penicillin sodium under an incubator with an atmosphere of $5 \%$ $\mathrm{CO}_{2} / 95 \%$ air at $37^{\circ} \mathrm{C}$.

\section{RNA interference}

The sequences of the siRNAs used to suppress NCK1-AS1 expression were sense 5'-GAAUGUCA UCCCAGCCGAATT - ${ }^{\prime}$, antisense 5' -UUCGGCUGGG AUGACAUUCTT $-3^{\prime}$. The control siRNA sequence that targeted green fluorescent protein (GFP) were sense $5^{\prime}$ -UUCUCCGAACGUGUCACGUTT-3', antisense ${ }^{\prime}$ -ACGUGACACGUUCGGAGAATT-3'. The sequences of the short hairpin RNA used to suppress NCK1-AS1 expression were sense 5'-GATCCGAATGTCATCCC AGCCGAATTCAAGAGATTCGGCTGGGATGACATT CTTTTTTG-3', antisense 5'-AATTCAAAAAAGAA TGTCATCCCAGCCGAATCTCTTGAATTCGGCTGG
GATGACATTCG-3'. siRNAs were obtained from GenaPharma (Shanghai, China). siRNA and Lentviral3-GFPshRNA specfilly targeting NCK1-AS1 and negative control were designed and synthesized by Genepharma (Shanghai, China). Transfection of siRNA $(60 \mu \mathrm{M})$ was carried out using Lipofectamine ${ }^{\circ}$ RNAiMAX Reagent (Invitrogen) according to the manufacturer's instructions. Lent virus3-GFP-shRNA was used to infect CaSki cells. After infection $24 \mathrm{~h}$, CaSki cells were cultured in complete media containing puromycin $(3 \mu \mathrm{g} / \mathrm{ml})$ to generate a stable knockdown NCK1-AS1 cell line.

\section{Quantitative real-time PCR}

The following primer sequences were used $\rightarrow$ to detec$\mathrm{t} \rightarrow$ each gene: $5^{\prime}$-GGAGCGAGATCCCTCCAAAAT $-3^{\prime}$ and $5^{\prime}$-GGCTGTTGTCATACTTCTCATGG $-3^{\prime}$ for GAPDH, $5^{\prime}-$ TTCCCATTTCTCCCAGGTCC $-3^{\prime}$ and $5^{\prime}$ -TGGTTACTTTGAGCCTGCCT-3' for NCK1-AS1, 5' - AGAGTGGTGGAAATGCAGGA- ${ }^{\prime}$ and $5^{\prime}$-TTGA TGCCTGGTG ACTTTGC- $3^{\prime}$ for NCK1, $5^{\prime}$-TGGCA GCAGTACCAATGGC $-3^{\prime}$ and $5^{\prime}$-CCAGGTAGTCCT GTCAGAACTT $-3^{\prime}$ for SP1, 5'-CCAGGAGTTACTT CTATGCCTGA $-3^{\prime}$ and $5^{\prime}$-TTCATCCAGGGGAGGT ACAAC $-3^{\prime}$ for CDK2, 5'-AAACTACAGGTCAAGT GGTAGCC $-3^{\prime}$ and $5^{\prime}$-TCCTGCATAAGCACATCCT GA-3' for CDK1. miRNA reverse-transcription and the expression levels was performed by miRNA First Strand cDNA Synthesis Tailing Reaction Kit (B532451,Sangon Biotech, Shanghai, China) according to the manufacturer's instruction. Universal miRNA qRT-PCR Primer 5'-AACGAGACGACGACAGAC-3', 5'-GCAAATT CGTGAAGCGTTCCATA-3' for RNU6, 5'-TGGG GATTGGGTCAGGCC-3' for has-miR-6857, 5'-TAAT AG CTCAGAATGTCAGTTCTG-3' for hsa-miR-7705, 5'-ATGGGGACAGGGATC AGCAT-3' for hsa-miR6810. The reactions were performed using the SYBR Premix Ex TaqTM II (TIi RNaseH Plus,TAKARA), and the CFX Connect Real Time System (BIO-RAD) was used for analysis. Relative expression was normalized to the endogenous control GAPDH or RNU6 using the $2^{-\Delta \Delta \mathrm{Ct}}$ method.

\section{Cell proliferation and invasion assays in vitro}

Cell proliferation ability was determined by Cell counting Kit-8 (CCK8) (Dojindo Laboratories,Japan) after siRNA transient transfection for 24,48 and $72 \mathrm{~h}$. Colony formation assays were performed to monitor the cloning capability of stable knockdown NCK1-AS1 CC cells cloning capability. Two weeks later, colonies were fixed with methanol and stained by $0.25 \%$ crystal violet staining solution. For the invasion assay, cells were serum-starved overnight and $2 \times 10^{4}$ cells were seeded in a Matrigelcoated chamber and cultured for $48 \mathrm{~h}$. The invaded or migrated cells were fixed with $70 \%$ methanol and stained 
$0.25 \%$ crystal violet staining solution. Cells invading to the lower surface of filters were counted in five randomly selected fields. All experiments were carried out in triplicate.

\section{Tumor growth assay in vivo}

The animal study protocol was approved by the Animal Experimentation Ethics Committee of Chongqing Medical University. Six female Balb/c nude mice (aged four weeks) were provided by Beijing Laboratory Animal Research Center (Beijing, China) and housed in a pathogen-free animal facility (Laboratory Animal Center of Chongqing medical university). Briefly, six nude mice were randomly assigned to the control or experimental group (three mice per group). $1 \times 10^{6} \mathrm{shCtrl}$ and shNCK1-AS1 CaSki cells were suspended in $0.14 \mathrm{ml}$ of phosphate buffer solution and subcutaneously injected into the femoral area of nude mice. The tumor was measured with calipers and the volume was calculated using the formula: $(\pi / 6) x^{3}$, where $x=$ the largest diameter. Thirty-five days after tumor inoculation, the mice were sacrificed and the tumors were extracted to determine tumor weight. Data are presented as the mean $\pm \mathrm{SD}$.

\section{Fluorescence in situ hybridization analysis}

Cy3 labeled FISH probes were designed and synthesized by Genepharma (Shanghai, China). Cells were grown on glass coverslips, fixed with ice-cold 4\% paraformaldehyde for $20 \mathrm{~min}$, and blocked with Pre-hybridization Buffer for $30 \mathrm{~min}$. Cells were incubated with $25 \mu \mathrm{M}$ NCK1-AS1 FISH probe in hybridization buffer in Hybridization Buffer in the dark at $37^{\circ} \mathrm{C}$ overnight, washed three times with $2 \times$ SSC, incubated with DAPI for detection for $15 \mathrm{~min}$ according Fluorescent In Situ Hybridization Kit manufacturer (Ribo, Guangzhou, China), and then analyzed using a confocal fluorescence microscope (FV1000-D, Olympus, Tokyo, Japan).

\section{Luciferase reporter assay}

The SP1 binding motif in the promoter region of NCK1-AS1 was identified by JASPAR (http://jaspar. genereg.net/). The full-length and the different fragment sequences were synthesized and then cloned into the pGL3-basic vector (Promega, Madison). Cells were seeded into 24-well plates and then cotransfected with $100 \mathrm{ng}$ of pmirGLO-CDK1-WT, pmirGLO-CDK1-mut, and 200 $\mathrm{nmol} / \mathrm{L}$ of miR-6857 mimics or control by using Lipofectamine 2000. Luciferase assays were performed $48 \mathrm{~h}$ after transfection using the dual-luciferase reporter assay system (Promega). Luciferase activities were assessed using the Dual Luciferase Assay Kit (Promega), according to the manufacturer's instructions.

\section{Statistical analysis}

IBM SPSS statistics 23 was used for statistical analysis of cell invasion, colony formation, and tumor formation in the mice and conducted using the Student $t$ test. The association between NCK1-AS1 expression and tumor stage was determined by Chi-square test. $P \leq 0.05$ was considered as statistically significant.

\section{Acknowledgements}

This work was supported by the National Natural Science Foundation of China (Nos. 20125503110012 and cstc2011jjA10035).

\section{Author details}

${ }^{1}$ Molecular Medicine and Cancer Research Center, Chongqing Medical University, Chongqing 400016, China. ${ }^{2}$ Department of Biochemistry and Molecular Biology, Chongqing Medical University, Chongqing 400016, China. ${ }^{3}$ The Second Clinical College of Chongqing Medical University, Chongqing Medical University, Chongqing 400016, China

\section{Author contributions}

H.L., G.L., and F.S. conceived of the study, and participated in experiment design and coordination and they wrote the draft of the manuscript. Y.J. and J. C. performed the retrieval of data and statistical analysis. All authors read and approved the final manuscript.

\section{Conflict of interest}

The authors declare that they have no conflict of interest.

\section{Publisher's note}

Springer Nature remains neutral with regard to jurisdictional claims in published maps and institutional affiliations.

Supplementary Information accompanies this paper at https://doi.org/ 10.1038/s41419-017-0249-3.

Received: 21 July 2017 Revised: 19 December 2017 Accepted: 20 December 2017

Published online: 07 February 2018

\section{References}

1. McGuire, S. World Cancer Report 2014. Geneva, Switzerland: World Health Organization, International Agency for Research on Cancer, WHO Press, 2015. Adv. Nutr. 7, 418-419 (2016).

2. Smith, R. A., Brooks, D., Cokkinides, V., Saslow, D. \& Brawley, O. W. Cancer screening in the United States, 2013: a review of current American Cancer Society guidelines, current issues in cancer screening, and new guidance on cervical cancer screening and lung cancer screening. Cancer J. Clin. 63, 88-105 (2013).

3. Kim, J. J. et al. Inefficiencies and high-value improvements in U.S. cervical cancer screening practice: a cost-effectiveness analysis. Ann. Intern. Med. 163, 589-597 (2015).

4. Tewari, K. S. et al. Improved survival with bevacizumab in advanced cervical cancer. N. Engl. J. Med. 370, 734-743 (2014)

5. Canavan, T. P. \& Doshi, N. R. Cervical cancer. Am. Fam. Phys. 61, 1369-1376 (2000).

6. Moore, K. N. \& Rowland, M. R. Treatment advances in locoregionally advanced and stage ivb/recurrent cervical cancer: can we agree that more is not always better? J. Clin. Oncol.: Off. J. Am. Soc. Clin. Oncol. 33, 2125-2128 (2015).

7. Kapranov, P. et al. RNA maps reveal new RNA classes and a possible function for pervasive transcription. Science 316, 1484-1488 (2007).

8. Carninci, P. et al. The transcriptional landscape of the mammalian genome. Science 309, 1559-1563 (2005).

9. Hon, C. C. et al. An atlas of human long non-coding RNAs with accurate 5' ends. Nature 543, 199-204 (2017). 
10. Williamson, L. et al. UV irradiation induces a non-coding RNA that functionally opposes the protein encoded by the same gene. Cell 168, 843-855 (2017). e813.

11. llott, N. E. et al. Corrigendum: long non-coding RNAs and enhancer RNAs regulate the lipopolysaccharide-induced inflammatory response in human monocytes. Nat. Commun. 6, 6814 (2015).

12. $Y u, X$. et al. Long non-coding RNA Linc-RAM enhances myogenic differentiation by interacting with MyoD. Nat. Commun. 8, 14016 (2017).

13. Necsulea, A. et al. The evolution of IncRNA repertoires and expression patterns in tetrapods. Nature 505, 635-640 (2014).

14. Fatica, A. \& Bozzoni, I. Long non-coding RNAs: new players in cell differentiation and development. Nat. Rev. Genet. 15, 7-21 (2014).

15. Beermann, J., Piccoli, M. T., Viereck, J. \& Thum, T. Non-coding RNAs in development and disease: background, mechanisms, and therapeutic approaches. Physiol. Rev. 96, 1297-1325 (2016).

16. Knoll, M., Lodish, H. F. \& Sun, L. Long non-coding RNAs as regulators of the endocrine system. Nat. Rev. Endocrinol. 11, 151-160 (2015).

17. Geisler, S. \& Coller, J. RNA in unexpected places: long non-coding RNA functions in diverse cellular contexts. Nat. Rev. Mol. Cell. Biol. 14, 699-712 (2013).

18. Somarowthu, S. et al. HOTAIR forms an intricate and modular secondary structure. Mol. Cell. 58, 353-361 (2015).

19. Yoon, J. H. et al. Scaffold function of long non-coding RNA HOTAIR in protein ubiquitination. Nat. Commun. 4, 2939 (2013)

20. Niknafs, Y. S. et al. The IncRNA landscape of breast cancer reveals a role for DSCAM-AS1 in breast cancer progression. Nat. Commun. 7, 12791 (2016)

21. Kogo, R. et al. Long noncoding RNA HOTAIR regulates polycomb-dependent chromatin modification and is associated with poor prognosis in colorectal cancers. Cancer Res. 71, 6320-6326 (2011).

22. Denisenko, O., Shnyreva, M., Suzuki, H. \& Bomsztyk, K. Point mutations in the WD40 domain of Eed block its interaction with Ezh2. Mol. Cell Biol. 18, 5634-5642 (1998).
23. Katayama, S. et al. Antisense transcription in the mammalian transcriptome Science 309, 1564-1566 (2005).

24. Gupta, R. A. et al. Long non-coding RNA HOTAIR reprograms chromatin state to promote cancer metastasis. Nature 464, 1071-1076 (2010).

25. Fu, W. M. et al. Hotair mediates hepatocarcinogenesis through suppressing miRNA-218 expression and activating P14 and P16 signaling. J. Hepatol. 63, 886-895 (2015).

26. Costanzi, C. \& Pehrson, J. R. Histone macroH2A1 is concentrated in the inactive X chromosome of female mammals. Nature 393, 599-601 (1998).

27. Wutz, A. \& Gribnau, J. X. inactivation Xplained. Curr. Opin. Genet. Dev. 17, 387-393 (2007).

28. Faghihi, M. A. et al. Expression of a noncoding RNA is elevated in Alzheimer's disease and drives rapid feed-forward regulation of beta-secretase. Nat. Med. 14, $723-730$ (2008).

29. Tufarelli, C. et al. Transcription of antisense RNA leading to gene silencing and methylation as a novel cause of human genetic disease. Nat. Genet. 34, 157-165 (2003).

30. Sun, N. X. et al. Long noncoding RNA-EBIC promotes tumor cell invasion by binding to $\mathrm{EZH} 2$ and repressing E-cadherin in cervical cancer. PLOS ONE $\mathbf{9}$ e100340 (2014).

31. Prensner, J. R. \& Chinnaiyan, A. M. The emergence of IncRNAs in cancer biology. Cancer Discov. 1, 391-407 (2011).

32. Qi, P. \& Du, X. The long non-coding RNAs, a new cancer diagnostic and therapeutic gold mine. Mod. Pathol.: Off. J. US Can. Acad. Pathol. Inc. 26, 155-165 (2013).

33. Mitra, S. A., Mitra, A. P. \& Triche, T. J. A central role for long non-coding RNA in cancer. Front. Genet. 3, 17 (2012).

34. Yuan, J. H. et al. A long noncoding RNA activated by TGF-beta promotes the invasion-metastasis cascade in hepatocellular carcinoma. Cancer Cell. 25, 666-681 (2014). 